\title{
Anti-inflammatory effects of pre-seasonal Th I-adjuvant vaccine to Parietaria judaica in asthmatics
}

This article was published in the following Dove Press journal:

Journal of Asthma and Allergy

23 March 20II

Number of times this article has been viewed

\section{Nicola Scichilone \\ Chiara Minaldi \\ Roberta Santagata \\ Salvatore Battaglia \\ Gaetana Camarda \\ Vincenzo Bellia}

Dipartimento Biomedico di Medicina Interna e Specialistica (Di.Bi.M.I.S.), Sezione di Pneumologia, University of Palermo, Palermo, Italy
Correspondence: Nicola Scichilone Dipartimento Biomedico di Medicina Interna e Specialistica (Di.Bi.M.I.S.), Sezione di Pneumologia, University of Palermo, via Trabucco I80, 90 I 46 Palermo, Italy Tel +39 09l-6802655 Fax +3909I-6882842 Email n.scichilone@libero.it
Background: The ultra-short course pre-seasonal allergy vaccine, containing appropriate allergoids with the adjuvant monophosphoryl lipid A (MPL), may be effective in treating allergic symptoms.

Objective: To explore the timing of the immunological responses to the pre-seasonal allergy vaccine.

Methods: Four subcutaneous injections of the active product (Pollinex Quattro) were administered to 20 Parietaria-sensitive intermittent asthmatics (M/F: 12/8; age: $48 \pm 10$ years; $\mathrm{FEV}_{1} \%$ predicted: $108 \% \pm 12 \%$ ) during the 6 weeks prior to the start of the pollen season. Exhaled breath condensate (EBC) was collected immediately before the first and immediately after the last injections $\left(\mathrm{t}_{1}\right.$ and $\left.\mathrm{t}_{2}\right)$, during the pollen season $\left(\mathrm{t}_{3}\right)$ and after $\left(\mathrm{t}_{4}\right)$ the pollen season. EBC was analyzed to determine the levels of $\mathrm{pH}$ and 8-isoprostane. Ten Parietaria-sensitive asthmatics served as the untreated control group at $\mathrm{t}_{1}$ and $\mathrm{t}_{2}$.

Results: Measured $\mathrm{pH}$ levels were $7.64 \pm 0.33$ at $\mathrm{t}_{1}, 7.67 \pm 0.23$ at $\mathrm{t}_{2}, 7.72 \pm 0.34$ at $_{3}$, and $7.82 \pm 0.34$ at $_{4}(P=0.049$ vs baseline $)$. 8-isoprostane levels were significantly lower than baseline at each visit (mean difference from baseline, for $\mathrm{t}_{2}:-0.77 \mathrm{pg}, P=0.031$; for $\mathrm{t}_{3}$ : $-0.92 \mathrm{pg}$, $P=0.010$; for $\mathrm{t}_{4}:-0.70 \mathrm{pg}, P=0.048$ ). In the control group, $\mathrm{pH}$ levels were $7.73 \pm 0.26$ at baseline and did not change after 6 weeks $(7.79 \pm 0.25, P=0.33)$. Similarly, the concentrations of 8-isoprostane in the control group were not different from those of the study group at baseline $(P=0.86)$, and the levels remained unchanged after 6 weeks $(P=0.58)$.

Conclusion: These findings show that the ultra-short course of vaccine adjuvated with MPL acutely reduces the degree of airway inflammation, as expressed by markers of oxidative stress, and suggest that this reduction is maintained during and after the pollen season.

Keywords: allergen, asthma, immunotherapy, inflammation, pollen, exhaled breath condensate

\section{Introduction}

The therapeutic approach to allergic asthma is 3-fold: avoidance, medications, and immunotherapy. Avoidance of the specific allergen that is responsible for the respiratory symptoms, although ideal, is not always possible given that some pollens are almost permanently present in the air; on the other hand, the efficacy of allergen avoidance may be controversial, as in the case of indoor allergens. Pharmacological therapy is used to treat airway inflammation and obstruction. As established by current guidelines, ${ }^{1}$ immunotherapy is advocated as an additional tool in the mildest forms of allergic asthma. Immunotherapy has been demonstrated to delay the occurrence of worsening asthma, ${ }^{2}$ to prevent new allergen sensitizations from occurring, ${ }^{3,4}$ and to reduce the frequency and severity of respiratory symptoms. ${ }^{5,6}$ 
Two types of immunotherapy have been developed in the past and are currently used in clinical practice. The subcutaneous allergy injections (SCIT) are administered at gradually increasing doses until the maintenance dose is attained. Sublingual immunotherapy (SLIT) has been used extensively in Europe as an alternative to SCIT. Optimal dosing, standardization, and control of the products, and allergen modifications such as the introduction of allergoids (chemically modified allergens) have drammatically improved the safety and tolerability of immunotherapy.

To improve the efficacy of immunotherapy, immunological adjuvants have been incorporated in the within allergy vaccines. The monophosphoryl lipid A (MPL), a detoxified lipopolysaccharide component extracted and purified from Salmonella minnesota, has been demonstrated to promote and increase the immunological response. ${ }^{7}$ The new MPL vaccine has been demonstrated to be effective in reducing nasal and ocular symptom scores, skin test sensitiviy, and medication score. ${ }^{8}$ Little is known, however, about the vaccine's efficacy in treating asthma. Despite the clinical advantages of this product, ${ }^{9}$ the immunological changes following treatment have not been fully elucidated. In addition, the changes induced by the MPL-adjuvated vaccine on lower airway inflammation are not clear. We reasoned that the addition of MPL would enhance the anti-inflammatory properties of the vaccine. Based on these assumptions, shorter courses of immunotherapy have been administered, resulting in clinical and functional improvements. However, whether these impovements are related to a reduction in the severity of airway inflammation is not known. We designed our study to assess the role of subcutaneous MPL-adjuvated vaccine on features of airway inflammation in intermittent asthmatics who were sensitized to Parietaria judaica using the exhaled breath condensate (EBC) methodology.

\section{Methods}

\section{Subjects}

We recruited individuals attending the Allergy Outpatient Clinic of the Division of Respiratory Diseases of the University of Palermo, Italy, who had received the diagnosis of intermittent asthma. The main criteria for inclusion in the current study were: 1) skin test positivity only to Parietaria, 2) no history of any type of specific immunotherapy. Subjects who were also sensitized to other allergens or were under regular anti-asthmatic treatment were excluded from the study. Short-acting $\beta$-agonists were withheld for at least 12 hours and anti-histamines for 48 hours prior to each evaluation.
Subjects were tested at least 4 weeks after their most recent upper respiratory infection. Ten individuals with intermittent asthma who were allergic only to Parietaria served as untreated controls. Both the study and the control groups received symptomatic therapy for upper and lower respiratory symptoms as required. The study was approved by the local ethics committee and all subjects gave written, informed consent.

\section{Study design}

The study had a longitudinal experimental design and consisted of three phases. Figure 1 describes the protocol and the time-frame of the study. A total of 7 visit days were needed to complete the study.

\section{Phase I}

At the first visit $\left(\mathrm{t}_{0}\right)$, which took place in June and July, all inclusion and exclusion criteria were checked and subjects were enrolled. The screening evaluation included a respiratory questionnaire for both upper and lower airways, allergy skin testing to a panel of 10 common aeroallergens, and a complete functional assessment. The evaluation of the nasal involvement was also performed according to the Allergic Rhinitis and its impact on Asthma (ARIA) document recommendations. ${ }^{10}$ Spirometric testing was performed with a water-sealed spirometer (Biomedin, Padova Italy). Acceptability and reproducibility of $\mathrm{FEV}_{1}$ and forced vital capacity (FVC) were evaluated based on the recommendations of the European Respiratory Society. ${ }^{11}$

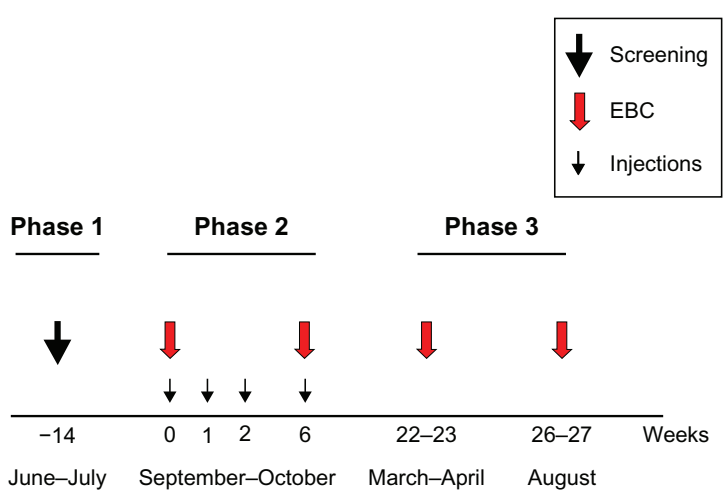

Figure I Schematic of the protocol that describes the study (see also study design). A total of 7 visit days were needed to complete the study. Phase I was the screening evaluation consisting of lung function assessment and skin prick testing. Phase 2 consisted of 4 visits at which the study subjects received subcutaneous injections of the active product with increasing strength. In the first week (week 0 ) and the last (week 6) the allergy vaccine was injected, and the biological assessment was conducted. The untreated control group also participated in this phase. Phase 3 was only conducted in the study subjects. In March and April (during pollen season) and August (after pollen season) each subject repeated the biological examination. Abbreviation: EBC, exhaled breath condensate. 


\section{Phase 2}

During September-October (pre-pollen season), each subject visited the laboratory on 4 separate occasions at which time they received a subcutaneous injection of the active product with increasing strength. The first three $1.0 \mathrm{~mL}$ increasing strength injections were administered at 1 -week intervals, and the last top-strength injection was administered at 6 weeks from the first injection. At the time of the first $\left(\mathrm{t}_{1}\right)$ and the last $\left(t_{2}\right)$ injections of the allergy vaccine, functional and biological assessments were conducted. The ultra-short course pre-seasonal allergy vaccine (Pollinex ${ }^{\circledR}$ Quattro, Allergy Therapeutics, Worthing, England), containing appropriate allergoids with MPL, was employed. The vaccine consists of purified extract of Parietaria judaica pollen allergen (par j1: $16.64 \mu \mathrm{g} / \mathrm{mL}$ ), which is modified with glutaraldehyde. The MPL adjuvant was adsorbed to the L-tyrosine at $50 \mu \mathrm{g} / \mathrm{mL}$ in all vaccine dose levels. The 10 asthmatics who served as untreated controls underwent EBC collection twice during the study at a 6-week interval.

The biological assessment consisted of evaluating the inflammatory pattern of the lower airways which was established through EBC. EBC was collected (EcoScreen, Jaeger, Germany) as the patient breathed room-air through a mouthpiece connected to a 2-way non-rebreathing valve and to a saliva trap. The valve was connected to an add-on unit (ECoVent, Jaeger, Germany), to standardize EBC collection. The device (ECoVent) measured breathing volume and the procedure was stopped at $120 \mathrm{~L}$ of total volume, which allowed collection of about $2 \mathrm{~mL}$ of condensate from the patient in 10 to 15 minutes, depending on the patient's breathing frequency. Each patient was asked to breath at a normal frequency and tidal volume wearing a nose clip, after rinsing their mouth with water. The collection was performed from 09.00 to 14.00 hours in the morning. The collected fluid was immediately subdivided into $300 \mu \mathrm{L}$ aliquots in Eppendorf tubes, which were directly transferred to $-70^{\circ} \mathrm{C}$ pending analysis.

\section{$\mathrm{pH}$ measurement}

The EBC $\mathrm{pH}$ was measured using a $\mathrm{pH}-$ meter with a micro-electrode (pH-221 Hanna Instruments, Leighton, $\mathrm{UK})$. The $\mathrm{pH}$-meter range was -2.00 to 16.00 with a mean resolution/accuracy of the order of $0.01 \pm 0.02$. A stable $\mathrm{pH}$ was achieved after de-areation of the EBC specimens by bubbling them with nitrogen at 2 bar for 5 minutes. ${ }^{12}$ All samples were analyzed together and each sample was tested in duplicate.

\section{8-Isoprostane measurement}

8-Isoprostane concentrations in EBC were measured with a specific enzyme immunoassay (EIA) kit (Cayman Chemical, Ann Arbor, MI). The antiserum used in the EIA has 100\% cross-reactivity with 8 -isoprostane and $20.6 \%$ cross-reactivity with prostaglandin $\mathrm{F}_{2 \alpha}\left(\mathrm{PGF}_{2 \alpha}\right), 4 \%$ cross-reactivity with $\mathrm{PGF}_{3 \alpha}, 1.84 \%$ with $\mathrm{PGE}_{2}$ and $1.7 \%$ cross-reactivity with $\mathrm{PGF}_{1 \alpha}$. The detection limit of the assay is $2.7 \mathrm{pg} / \mathrm{mL}$. All samples were analyzed together and each sample was tested in duplicate.

\section{Phase 3}

As a secondary aim, the biological evaluation performed in Phase 2 was repeated in the study group during the pollen season (March-April, $\mathrm{t}_{3}$ ), and after pollen season (August, $\mathrm{t}_{4}$ ).

\section{Statistical analysis}

The $\mathrm{pH}$ and the 8-Isoprostane concentrations in the EBC represent the outcomes of the study. Data are presented as mean \pm SD. Treatment-induced changes from baseline over time were analyzed by repeated measures one-way ANOVA, followed by appropriate post hoc tests. Linear regressions were used to examine the relationship between the biological variable and indices of lung function before the beginning of the treatment. Significance was assumed for two-tailed values of $P \leq 0.05$.

\section{Results \\ Phase I}

A total of 20 intermittent asthmatics (M/F: 12/8, age: $48 \pm 10$ years) took part in the study. As expected, lung function was in the normal range $\left(\mathrm{FEV}_{1} / \mathrm{FVC}\right.$ : $0.84 \pm 0.02$; $\mathrm{FEV}_{1} \%$ predicted: $108 \% \pm 12 \%$ ). The asthmatics who served as the control group (M/F: $5 / 5)$, were similar to the study group in age ( $42 \pm 8$ years, $P=0.76)$ and in lung function characteristics $\left(\mathrm{FEV}_{1} / \mathrm{FVC}: 0.88 \pm 0.01 ; \mathrm{FEV}_{1} \%\right.$ predicted: $114 \% \pm 9 \%$ ). Table 1 describes the percentage of subjects

Table I Clinical characteristics of subjects belonging to the study and the control groups related to the 4 weeks preceding the time of recruitment

\begin{tabular}{lll}
\hline & $\begin{array}{l}\text { Study } \\
\text { group }\end{array}$ & $\begin{array}{l}\text { Control } \\
\text { group }\end{array}$ \\
\hline Upper respiratory symptoms (\% of subjects) & 40 & 50 \\
Lower respiratory symptoms (\% of subjects) & 20 & 10 \\
Use of short-acting $\beta$-agonists (\% of subjects) & 20 & 10 \\
Use of antihistamines (\% of subjects) & 25 & 15 \\
\hline
\end{tabular}

Note: Data are expressed as percentages of subjects who experienced symptoms or required medications. 
from both groups who experienced upper and/or lower symptoms at the time of recruitment, and who required rescue medications. The four asthmatics of the study group and the only control subjects who had lower respiratory symptoms in the weeks that preceded the beginning of the study were also suffering from upper airway symptoms.

\section{Phase 2}

None of the volunteers reported any adverse events associated with the immunotherapy. During this phase, none of the subjects required rescue medications or anti-histamines. Treatment with pre-seasonal adjuvated vaccine did not significantly change the EBC pH $\left(7.64 \pm 0.33\right.$ at $\mathrm{t}_{1}$ and $7.67 \pm 0.23$ at $\mathrm{t}_{2} ; P=0.52$ ). In the control group the $\mathrm{pH}$ was $7.73 \pm 0.26$ and did not change at the following assessment performed 6 weeks later $(7.79 \pm 0.25, P=0.33)$. The concentrations of 8-isoprostane in the EBC significantly dropped immediately after the ultra-short course of allergy vaccine $\left(\mathrm{t}_{2}\right)$ : the mean difference from baseline was $-0.77 \mathrm{pg}$ $(P=0.031)$. The baseline $\left(\mathrm{t}_{1}\right)$ and post treatment $\left(\mathrm{t}_{2}\right)$ values for each individual are depicted in Figure 2. In the control group, the concentrations of 8-isoprostane were not different from those of the study group at baseline $(44.1 \pm 1.4 \mathrm{pg} / \mathrm{mL}$ vs $44.4 \pm 0.5 \mathrm{pg} / \mathrm{mL}, P=0.86$; study group vs controls), and remained unchanged after 6 weeks $(44.6 \pm 1.2 \mathrm{pg} / \mathrm{mL}$, $P=0.58)$. This finding lends support to the occurrence of the early anti-inflammatory effect of immunotherapy in the study group.

The concentrations of 8-isoprostane and $\mathrm{pH}$ at $\mathrm{t}_{1}$ were not associated with baseline lung function as expressed by $\mathrm{FEV}_{1} \%$ predicted $(r=0.31, P=0.23$ and $r=0.02, P=0.91$, respectively).

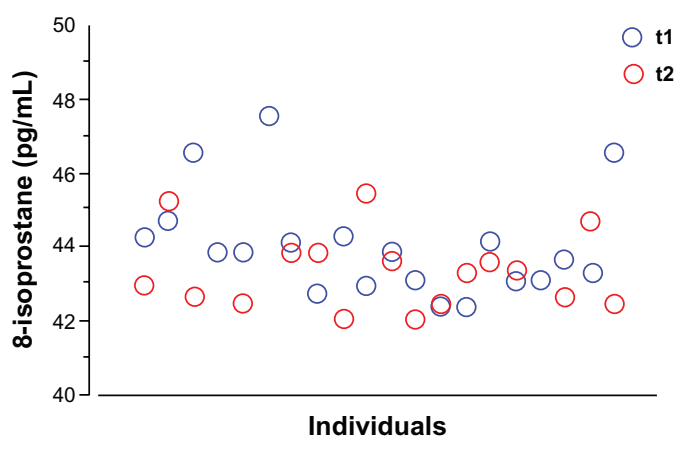

Figure 2 Individual data for the concentrations of 8-isoprostane assessed in the exhaled breath condensate of the study subjects. The blue circles represent the levels of 8-isoprostane measured before the first injection of the vaccine $\left(t_{1}\right)$, and the red circles represent the levels of 8-isoprostane measured immediately after the last injection of the vaccine $\left(t_{2}\right)$. Each individual ( $x$ axis) is expressed by a blue circle and the respective red circle.

Abbreviation: pg, picograms.

\section{Phase 3}

As a secondary aim, we tested whether changes in markers of airway inflammation also occurred in the study subjects during and after pollen season. This phase had only an exploratory aim, and did not include the control group. All study subjects completed the study. Five subjects who experienced mild asthma exacerbations during the study, presumably due to respiratory viral infections, had their next respective assessment postponed for 3 weeks. The EBC $\mathrm{pH}$ increased in comparison to the baseline values, both at $t_{3}$ $(7.72 \pm 0.34)$ and $t_{4}(7.82 \pm 0.35)$, reaching a significantly higher level only at $\mathrm{t}_{4}$ with respect to baseline $(P=0.049)$ (Figure 3). Remarkably, the 8-isoprostane levels remained significantly lower than baseline during the allergy season (mean difference from baseline: $-0.92 \mathrm{pg}, P=0.010$ ). When the collection of EBC was performed outside the pollen season $\left(t_{4}\right)$, the concentrations of 8 -isoprostane remained lower than the baseline values (mean difference from baseline: $-0.70 \mathrm{pg}, P=0.048$ ) (Figure 4). As expected, lung function assessed outside pollen season did not differ from that recorded at the beginning of the study $\left(\mathrm{FEV}_{1} / \mathrm{FVC}\right.$ : $0.81 \pm 0.02 ; \mathrm{FEV}_{1} \%$ predicted: $\left.111 \% \pm 10 \%, P=0.81\right)$

\section{Discussion}

This is a small study demonstrating that an ultra-short course immunotherapy, consisting of 4 pre-seasonal injections with pollen allergoids formulated with MPL, exerts early antiinflammatory effects, as documented by the significant reduction in the levels of 8-isoprostane in the EBC. The findings of the study also suggest that the airway inflammatory changes are maintained during and after the pollen season. This is indicated by the significantly lower levels of 8-isoprostane

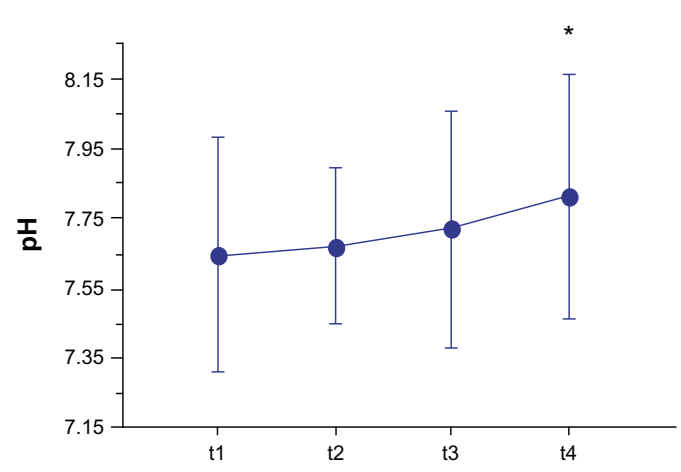

Figure 3 Means (connected circles) for the $\mathrm{pH}$ levels that were assessed in the exhaled breath condensate of the study subjects. These evaluations were obtained at the time of the first $\left(t_{1}\right)$ and the last $\left(t_{2}\right)$ pre-seasonal injections, during $\left(t_{3}\right)$ and outside $\left(\mathrm{t}_{4}\right)$ the pollen season.

Notes: Symbols indicate statistically significant effects of immunotherapy in post-hoc analyses: $* P=0.049, \mathrm{t}_{4}$ vs baseline. 


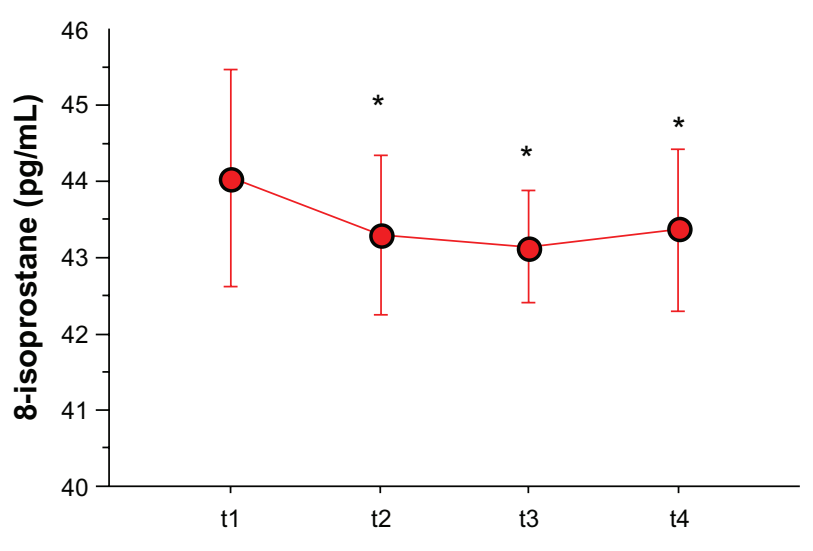

Figure 4 Means (connected circles) for the concentrations of 8-isoprostane that were assessed in the EBC of the study subjects. These evaluations were obtained at the time of the first $\left(t_{1}\right)$ and the last $\left(t_{2}\right)$ pre-seasonal injections, during $\left(t_{3}\right)$ and after $\left(\mathrm{t}_{4}\right)$ the pollen season.

Notes: Symbols indicate statistically significant effects of immunotherapy in post hoc analyses: $* P=0.031, \mathrm{t}_{2}$, vs baseline; $P=0.010, \mathrm{t}_{3}$ vs baseline; $P=0.048, \mathrm{t}_{4}$ vs baseline Abbreviations: $\mathrm{EBC}$, exhaled breath condensate; pg, picograms.

throughout the period of the study (during and after pollen season). These observations provide additional explanations for the clinical efficacy of the pre-seasonal immunotherapy.

The pre-seasonal vaccine employed in the current study consists of natural allergens combined through a "depot" technology to glutaraldehyde-modified pollens in order to offer higher tolerability. The immunological response is specifically powered by an adjuvant, 3-deacylated monophosphoryl lipid A (MPL), which is a purified, detoxified glucolipid extracted from the cell walls of Salmonella minnesota R595. ${ }^{13}$ MPL acts as an agonist of the toll-like 4 receptors (TLR4), which play a key role in the initial immune response against pathogens. ${ }^{14-16}$ It is plausible to speculate that the addition of MPL would account for the acute anti-inflammatory effects that were observed in the current study. However, the lack of a control group receiving vaccine with no MPL does not allow definite conclusions to be drawn in this respect.

The main finding of our study is the reduction in airway inflammation, as expressed by 8 -isoprostane concentrations in the EBC, following the ultra-short course of pre-seasonal immunotherapy. Isoprostanes are prostaglandin-like compounds formed from the free radical catalysed peroxidation of arachidonic acid. ${ }^{17,18}$ Contrary to the reactive oxygen species, which are unstable and short-lived, and as a consequence difficult to measure, 8-isoprostane is a stable product of lipid peroxidation, and is therefore a reliable marker of oxidative stress. ${ }^{19}$ Increased levels of 8 -isoprostane were found in EBC of asthmatic subjects, confirming the role of oxidative stress in the pathogenesis of asthma. ${ }^{20,21}$ Recently, Carraro and colleagues detected higher levels of 8-isoprostane in the $\mathrm{EBC}$ of asthmatic children with problematic asthma, suggesting that oxidative stress characterizes this asthmatic phenotype..$^{22}$ The concentrations of 8-isoprostane decreased after treatment with oral steroids, ${ }^{23}$ whereas they appear to be relatively resistant to inhaled corticosteroids..$^{24,25}$ To our knowledge, this is the first observation of a decrease in 8-isoprostane concentrations after adjuvated immunotherapy, which suggests its anti-inflammatory/antioxidant effects on lower airways. As previously stated, it is unclear whether this effect is specifically due to the properties of the adjuvant that is incorporated in the vaccine. Interestingly, this phenomenon occurred immediately after the series of vaccine injections, and the reduction persisted throughout pollen season and after pollen season. Further studies are needed to explore the timing of the immunological response to immunotherapy.

In the present study, we failed to demonstrate correlations between 8-isoprostane in $\mathrm{EBC}$ and lung function tests. This was relatively surprising, since previous observations from our group had shown that 8-isoprostane was inversely correlated with FEV ${ }_{1} \%$ predicted. ${ }^{26}$ However, these results are consistent with previous findings from Montuschi and colleagues ${ }^{20}$ and Zhao and colleagues. ${ }^{27}$ Discrepancies in this correlation are presumably due to differences in clinical or demographic characteristics of the study subjects, and/or different techniques used for EBC analysis. In the current study, inclusion criteria defined the subjects as intermittent asthmatics with lung function in the normal range; therefore, any significant correlation with indices of airway inflammation would seem unlikely.

Many authors have used the assessment of $\mathrm{pH}$ in the EBC as a measure of airway acidity in association with airways inflammation under different conditions. Hunt and colleagues demonstrated that $\mathrm{EBC}$ from patients with acute asthma showed a $\mathrm{pH}$ substantially lower than that recorded in healthy controls, and the levels of $\mathrm{pH}$ in asthma normalized with systemic steroid therapy. ${ }^{28}$ In a recently published longitudinal study, ${ }^{29}$ the acidification of EBC was associated with exacerbations of asthma. Furthermore, the condensate $\mathrm{pH}$ increased along with treatment. Interestingly, it has been demonstrated that EBC nitrite levels increase after 1-year immunotherapy. ${ }^{30}$ Hunt and colleagues argued that this increase is due to the effect of inhaled corticosteroid withdrawal that follows successful immunotherapy. However, an alternative hypothesis could be that higher $\mathrm{pH}$ levels induced by immunotherapy may lead to an increase in EBC nitrite, as already observed by Hunt and colleagues. ${ }^{28}$ In the 
current study, changes in the $\mathrm{pH}$ were small and not significant, except at the end of the study (after pollen season).

The study has several limitations. First, we included a control group that did not undergo a placebo treatment, nor were they followed during or after the pollen season. This limits the conclusions of the study. Indeed, the lack of a placebo injection, ie, administration of vaccine without MPL adjuvant, does not allow for definite conclusions on the contribution of the adjuvant to the efficacy of the vaccine. In addition, the lack of a control group during and outside the pollen season can only suggest, but not prove, that the pre-seasonal vaccine has a long-lasting effect on markers of airway inflammation. Second, we acknowledge that the EBC collection merits standardization so that its use in clinical practice is no longer a limitation.

The allergy vaccine containing MPL has been demonstrated to be safe and well-tolerated. Many studies involving MPL have shown a lack of toxic effects. ${ }^{8,9,31}$ From a clinical standpoint, efficacy of the Th-1 adjuvated vaccine has largely been demonstrated. ${ }^{8,9,32}$ It is logical to speculate that the requirement of only 4 pre-seasonal injections to achieve a satisfactory clinical response in terms of nasal, ocular, and bronchial symptoms is expected to dramatically improve adherence to treatment. In conclusion, our study indicates that the pre-seasonal adjuvated vaccine exerts acute inflammatory changes in the airways of allergic asthmatics. These findings could contribute to further explanation of the clinical benefit of the ultra-short pre-seasonal vaccine.

\section{Acknowledgment}

We would like to thank Timothy J Hoenig, MS, for his valued help in the writing of the manuscript.

\section{Disclosure}

The authors disclose no conflicts of interest.

\section{References}

1. Global Initiative for Asthma. GINA report. Global strategy for asthma management and prevention. www.ginasthma.org. Accessed December 15, 2008.

2. Moller C, Dreborg S, Ferdousi HA, et al. Pollen immunotherapy reduces the development of asthma in children with seasonal rhinoconjunctivitis (the PAT-study). J Allergy Clin Immunol. 2002;109(2):251-256.

3. Des Roches A, Paradis L, Menardo JL, et al. Immunotherapy with a standardized Dermatophagoides pteronyssinus extract. VI. Specific immunotherapy prevents the onset of new sensitizations in children. J Allergy Clin Immunol. 1997;99(4):450-453.

4. Pajno GB, Barberio G, De Luca F, et al. Prevention of new sensitizations in asthmatic children monosensitized to house dust mite by specific immunotherapy. A six-year follow-up study. Clin Exp Allergy. 2001; 31(9):1392-1397.
5. Bousquet J, Maasch H, Martinot B, et al. Double-blind, placebocontrolled immunotherapy with mixed grass-pollen allergoids. II. Comparison between parameters assessing the efficacy of immunotherapy. J Allergy Clin Immunol. 1988;82(3 Pt 1):439-446.

6. Hedlin G, Graff-Lonnevig V, Heilborn H, et al. Immunotherapy with cat- and dog-dander extracts. V. Effects of 3 years of treatment. J Allergy Clin Immunol. 1991;87(5):955-964.

7. McCormack PL, Wagstaff AJ. Ultra-short-course seasonal allergy vaccine (Pollinex Quattro). Drugs. 2006;66(7):931-938.

8. Drachenberg KJ, Wheeler AW, Stuebner P, et al. A well-tolerated grass pollen-specific allergy vaccine containing a novel adjuvant, monophosphoryl lipid A, reduces allergic symptoms after only four preseasonal injections. Allergy. 2001;56(6):498-505.

9. Gawchik SM, Saccar CL. Pollinex Quattro Tree: allergy vaccine. Expert Opin Biol Ther. 2009;9(3):377-382.

10. Passalacqua G, Durham SR. Allergic rhinitis and its impact on asthma update: allergen immunotherapy. JAllergy Clin Immunol. 2007;119(4): 881-891.

11. Pellegrino R, Viegi G, Brusasco V, et al. Interpretative strategies for lung function tests. Eur Respir J. 2005;26(5):948-968.

12. Horvath I, Hunt J, Barnes PJ, et al. Exhaled breath condensate: methodological recommendations and unresolved questions. Eur Respir J. 2005;26(3):523-548.

13. Hopkins M, Lees BG, Richardson DG, et al. Standardisation of glutaraldehyde-modified tyrosine-adsorbed tree pollen vaccines containing the Th1-inducing adjuvant, monophosphoryl lipid A (MPL). Allergol Immunopathol (Madr). 2001;29(6):245-254.

14. Martin M, Michalek SM, Katz J. Role of innate immune factors in the adjuvant activity of monophosphoryl lipid A. Infect Immun. 2003;71(5):2498-2507.

15. Wheeler AW, Marshall JS, Ulrich JT. A Th1-inducing adjuvant, MPL, enhances antibody profiles in experimental animals suggesting it has the potential to improve the efficacy of allergy vaccines. Int Arch Allergy Immunol. 2001;126(2):135-139.

16. Von Baehr V, Hermes A, von Baehr R, et al. Allergoid-specific T-cell reaction as a measure of the immunological response to specific immunotherapy (SIT) with a Th1-adjuvanted allergy vaccine. J Investig Allergol Clin Immunol. 2005;15(4):234-241.

17. Fam SS, Morrow JD. The isoprostanes: unique products of arachidonic acid oxidation-a review. Curr Med Chem. 2003;10(17):1723-1740.

18. Morrow JD, Harris TM, Roberts LJ. Noncyclooxygenase oxidative formation of a series of novel prostaglandins: analytical ramifications for measurement of eicosanoids. Anal Biochem. 1990;184(1): $1-10$.

19. Janssen LJ. Isoprostanes: an overview and putative roles in pulmonary pathophysiology. Am J Physiol. 2001;280(6):L1067-L1082.

20. Montuschi P, Corradi M, Ciabattoni G, et al. Increased 8-isoprostane, a marker of oxidative stress, in exhaled condensate of asthma patients. Am J Respir Crit Care Med. 1999;160(1):216-220.

21. Zanconato S, Carraro S, Corradi M, et al. Leukotrienes and 8-isoprostane in exhaled breath condensate of children with stable and unstable asthma. J Allergy Clin Immunol. 2004;113(2):257-263.

22. Carraro S, Cogo PE, Isak I, et al. EIA and GC/MS analysis of 8-isoprostane in EBC of children with problematic asthma. Eur Respir J. 2010;35(6):1364-1369.

23. Baraldi E, Carraro S, Alinovi R, et al. Cysteinyl leukotrienes and 8 -isoprostane in exhaled breath condensate of children with asthma exacerbations. Thorax. 2003;58(6):505-509.

24. Baraldi E, Ghiro L, Piovan V, et al. Increased exhaled 8-isoprostane in childhood asthma. Chest. 2003;124(1):25-31.

25. Mondino C, Ciabattoni G, Koch P, et al. Effects of inhaled corticosteroids on exhaled leukotrienes and prostanoids in children. J Allergy Clin Immunol. 2004;114(4):761-667.

26. Battaglia S, den Hertog H, Timmers MC, et al. Small airways function and molecular markers in exhaled air in mild asthma. Thorax. 2005; 60(8):639-644. 
27. Zhao JJ, Shimizu Y, Dobashi K, et al. The relationship between oxidative stress and acid stress in adult patients with mild asthma. J Investig Allergol Clin Immunol. 2008;18(1):41-45.

28. Hunt JF, Fang K, Malik R, et al. Endogenous airway acidification. Implications for asthma pathophysiology. Am J Respir Crit Care Med. 2000;161(3 Pt 1):694-699.

29. Antus B, Barta I, Kullmann T, et al. Assessment of exhaled breath condensate $\mathrm{pH}$ in exacerbations of asthma and COPD: a longitudinal study. Am J Respir Crit Care Med. 2010;182(12):1492-1497.

30. Inci D, Altintas DU, Kendirli SG, et al. The effect of specific immunotherapy on exhaled breath condensate nitrite levels. Allergy. 2006;61(7):899-900.
31. Baldrick P, Richardson D, Wheeler AW. Safety evaluation of a glutaraldehyde modified tyrosine adsorbed housedust mite extract containing monophosphoryl lipid A (MPL) adjuvant: a new allergy vaccine for dust mite allergy. Vaccine. 2001;20(5-6):737-743.

32. Rosewich M, Schulze J, Fischer von Weikersthal-Drachenberg KJ, Zielen S. Ultra-short course immunotherapy in children and adolescents during a 3-years post-marketing surveillance study. Pediatr Allergy Immunol. 2010;21(Pt 2):185-9.

\section{Publish your work in this journal}

The Journal of Asthma and Allergy is an international, peer-reviewed open-access journal publishing original research, reports, editorials and commentaries on the following topics: Asthma; Pulmonary physiology; Asthma related clinical health; Clinical immunology and the immunological basis of disease; Pharmacological interventions and

\section{Dovepress}

new therapies. Issues of patient safety and quality of care will also be considered. The manuscript management system is completely online and includes a very quick and fair peer-review system, which is all easy to use. Visit http://www.dovepress.com/testimonials.php to read real quotes from published authors.

Submit your manuscript here: http://www.dovepress.com/journal-of-asthma-and-allergy-journal 